\title{
Corela
}

Cognition, représentation, langage

HS-31 | 2020

Métalinguistiques.

\section{Aux lisières du métalinguistique : l'effet métalinguistique.}

L'exemple de Pas pleurer de Lydie Salvayre

\section{Catherine Rannoux}

\section{(2) OpenEdition}

\section{Journals}

Édition électronique

URL : http://journals.openedition.org/corela/11581

DOI : $10.4000 /$ corela. 11581

ISSN : 1638-573X

\section{Éditeur}

Cercle linguistique du Centre et de l'Ouest - CerLICO

\section{Référence électronique}

Catherine Rannoux, « Aux lisières du métalinguistique : l'effet métalinguistique. », Corela [En ligne], HS-31 | 2020, mis en ligne le 02 juillet 2020, consulté le 03 juillet 2020. URL: http:// journals.openedition.org/corela/11581; DOI : https://doi.org/10.4000/corela.11581

Ce document a été généré automatiquement le 3 juillet 2020

\section{(c) (1) (9)}

Corela - cognition, représentation, langage est mis à disposition selon les termes de la licence Creative Commons Attribution - Pas d'Utilisation Commerciale - Partage dans les Mêmes Conditions 4.0 International. 


\title{
Aux lisières du métalinguistique : l'effet métalinguistique.
}

\author{
L'exemple de Pas pleurer de Lydie Salvayre
}

Catherine Rannoux

1 L'activité métalinguistique suppose, par définition, la production d'une glose qui vient souligner, commenter ou reformuler un segment linguistique devenu, au moins momentanément, objet du dire, qu'il s'agisse de revenir sur les mots de la langue ou les mots du discours: le métalinguistique oscille ainsi entre les catégories du métalinguistique à proprement parler, du métadiscursif, du métaénonciatif, et de l'épilinguistique. L'objet de cet article est d'interroger les limites du métalinguistique qui sera pris ici comme un terme générique englobant les différentes catégories: on s'interrogera sur la possibilité d'un continuum entre des formes marquées présentant un commentaire explicite (modalisations autonymiques avec glose métaénonciative ; reformulations par traduction ou rectification notamment dans la relation interlocutive, de type métadiscursif ou épilinguistique), et des segments d'où a disparu tout commentaire explicite.

2 L'objet qui servira de support à la réflexion sur l'hypothèse d'un effet métalinguistique est un texte littéraire, le dernier roman publié à ce jour de Lydie Salvayre, Pas pleurer ${ }^{1}$, dont la caractéristique est de mettre en œuvre de façon démultipliée les faits d'hétérogénéité : hétérogénéité liée au dialogisme, hétérogénéité des langues puisque coexistent le français et l'espagnol, donnant naissance à ce que l'écrivaine appelle le «fragnol ». La narration fait alterner les voix de la narratrice française, de sa mère espagnole qui a passé son âge adulte en France et pratique un français inventif et hasardeux, le fragnol. Elle fait entendre aussi la voix de Bernanos dont sont cités de larges fragments des Grands Cimetières sous la lune ${ }^{2}$, et les échos dialogiques des discours politiques du temps qui saturent les diatribes des personnages de Diego le communiste ou de Josep l'anarchiste.

3 L'hypothèse envisagée ici est celle de l'existence d'une interprétation métalinguistique: il s'agit de considérer ce qui relève d'un simple effet métalinguistique en raison d'une absence de glose. Pour être pris en compte, cet effet 
doit être suscité contextuellement par des indices de divers ordres. D'une part, l'absence de glose peut être paradoxalement montrée et compensée, même de façon ténue, par une autre absence (absence ponctuelle de ponctuation qui crée un effet de citation mécanique). D'autre part, l'effet métalinguistique se fonde sur le jeu de l'équivoque de la langue, en particulier dans le passage d'une langue à l'autre dans les situations de contact de langues qui sont susceptibles de déclencher faux-amis, approximations et autres pataquès. On se trouve donc face à un objet pour le moins paradoxal: l'effet métalinguistique sans glose. Le problème que pose ce type d'objet réside dans sa définition même et dans la possible confusion qui le menace : confusion entre ce qui est de l'ordre du représenté (donc relevant bien du champ du métalinguistique) et ce qui est simplement manifeste (exclu a priori de ce champ). L'effet métalinguistique se tiendrait donc à la lisière du métalinguistique, dans un continuum entre le représenté et le manifeste.

4 Je définirai dans un premier temps le fragnol, langue hybride fictive, constituée d'hétérogénéité dans la mesure où elle fait entendre une langue sous une autre et appelle ainsi le commentaire métalinguistique. Dans un deuxième temps je proposerai l'observation d'un ensemble de formes prises par le métalinguistique dans le texte, pour essayer de voir enfin comment un élargissement est envisageable aux formes non glosées, relevant de l'effet métalinguistique.

Le fragnol, « une langue impure, [...] qui fait entrer de l'autre »

Dans un beau texte intitulé «Défense et illustration du fragnol », L. Salvayre (2015) définit ainsi la langue pratiquée par sa mère, Montse, immigrée espagnole arrivée en France pendant la guerre d'Espagne :

un idiome qui empruntait, luxueusement, au français et à l'espagnol, un idiome complétant l'autre, chamboulant l'autre, suppléant l'autre, ravivant l'autre, libérant l'autre, distrayant l'autre, poétisant l'autre, dévergondant l'autre, espagnolisant l'autre, ou le faisant trébucher, rien que pour jouer. Et réciproquement. [...] Une langue qui chaque jour s'invente et s'élucubre, qui défixe les mots, les décloue de leur bois, les détourne du sens dont ils sont prisonniers, une langue qui défait, à pic nommé, les expressions toutes faites, qui ouvre des issues et fait passer de l'air. [...] Une langue impure, extrêmement, et qui, mine de rien, fait entrer de l'autre, qui fait entrer de l'Espagne, qui fait entrer des espagnes, qui fait entrer des autrement-dire, et peut-être, du même coup, des autrement-penser, on s'élargit, on respire. (p. 154-155, 156)

7 L'hybridation est la caractéristique fondamentale du fragnol dont le nom, mot-valise formé par télescopage entre "français " et "espagnol», explicite le principe de construction fondé sur la rencontre inattendue de formes langagières apparentées à des systèmes distincts : l'hybridation est alors facteur d'hétérogénéité, dans la mesure où elle introduit de l'autre (autre langue) dans la langue et opère une opacification momentanée du discours qui la met en œuvre. Elle peut procéder par traduction littérale de l'espagnol (calques) :

(1) j'en avais la peau de poule (p. 55) [piel de gallina] ou plus fréquemment par francisation de mots espagnols :

(2) à lui faire ces déclarations maravilleuses que les enfants font à leur mère lorsqu'ils les sentent tristes et en manque d'arrosage (p. 205) [maravillosa]

(3) Peux-tu me rendre le service, me dit tout à coup ma mère, de faire désapparaître le sirop pour la toux qui est coloqué sur le frigo? Il me raccorde très néfastement dona Pura. (p. 202) [desaparecer ; colocar ; recordar] 
Cette pratique du télescopage opère non seulement dans la présence d'une langue dans l'autre, mais aussi dans le recours à des approximations : à-peu-près langagiers par confusion entre deux unités lexicales : le cadeau de mes soucis (p. 162), une tête de litotte (p. 135), ou mauvaise segmentation pouvant donner lieu à des agglutinations : une noix blanche (p. 77). L'altérité présente dans les formes langagières, par contamination d'autres formes, appelle pour ainsi dire le commentaire métalinguistique, qu'il s'agisse de traduire dans un français normatif la forme de fragnol, de la commenter sous l'un ou l'autre de ses aspects (esthétique, dénotatif, etc.). Pour l'instant, on se contentera de poser que le fragnol, par définition, est un puissant déclencheur du métalinguistique, il en est sans doute même un vecteur.

10 L'énonciation que met en scène le roman s'avère propice au déploiement des situations de communication décrites par Jakobson (cité par Authier-Revuz 1995: 11) comme aptes à entraîner le recours au métalangage : a) rencontre d'une langue étrangère, b) procès d'acquisition du langage, c) relation interlocutive. Ces trois situations sont ainsi commentées par J. Authier-Revuz (1995: 12) :

On notera que, ce que ces trois situations ont en commun, c'est que le mécanisme communicationnel y est affecté d'un facteur d'hétérogénéité, de non-un - celui de la pluralité des langues (a), et, dans une même communauté linguistique, celui de l'inégalité dans les stades d'appropriation du système (b) et celui de la différence, idio/socio-lectale entre les deux pôles de l'interlocution (c) - et même que le « recours au métalangage » y est saisi comme une réponse, et même en (b) et (c) comme un remède à un problème ou un dysfonctionnement de communication: c'est-à-dire que le « rappel du langage » que constitue le métalangage « de tous les jours » $y$ est associé [...] à ce que j'ai appelé de la non-coïncidence.

11 Le fragnol déclencheur de commentaires métalinguistiques

12 Dans PP, cette non-coïncidence s'explicite donc fréquemment par des commentaires métalinguistiques qui pointent la non conformité des pratiques langagières maternelles. Dans un commentaire intégré au discours de la narratrice, qui confirme là son statut de détentrice des normes du français académique, le recours au simple mot de "langue » pour désigner la pratique discursive maternelle suffit à déclencher une cascade de reprises métalinguistiques. Dans le cas présent, ce sont des boucles métaénonciatives qui sont suscitées par cette désignation dont la compréhension pertinente est supposée requérir un déploiement réflexif, confirmant que le fragnol ne va pas de soi, et en conséquence, vérifiant son aptitude à susciter le métalinguistique :

(4) Ma mère me raconte tout ceci dans sa langue, je veux dire dans ce français bancal dont elle use, qu'elle estropie serait plus juste, et que je m'évertue constamment à redresser. (p. 111-112)

13 Il s'agit donc dans un premier temps de spécifier le sens requis, en recourant à une périphrase qui élimine le risque de confusion avec un autre sens possible (la langue maternelle de Montse, l'espagnol) et explicite le jugement normatif à l'encontre de la pratique en question, ce qui a pour effet de déclencher une nouvelle boucle énonciative pratiquant une "quasi-rature $»^{3}$ au profit d'un mot plus juste, "presque dit», au conditionnel. Ainsi est établie une partition entre le bancal et l'estropié, d'une part, et le redressement et la vertu d'autre part, entre l'inventivité du fragnol et l'académisme du français normatif.

14 Au-delà des considérations générales sur le fragnol comme langue, la pratique discursive déclenche également dans son cours les commentaires métalinguistiques, émis soit par la narratrice en tant qu'interlocutrice, soit par Montse comme locutrice 
de ce fragnol. On retrouve là la situation interlocutive décrite par Jakobson comme favorable à la production métalinguistique.

Le commentaire peut se faire minimal, visant à la rectification d'une forme jugée fautive, sans autre contenu que la substitution de la forme fautive. Ce sont notamment les cas de traduction comme dans l'extrait suivant où dans la continuité du récit narratorial s'emboitent deux Discours Directs (dorénavant DD), celui de Montse adolescente avec sa propre mère, mis en scène dans celui de Montse destiné à la narratrice :

(5) Ma grand-mère le remercie comme s'il la félicitait, mais moi, me dit ma mère, cette phrase me rend folle, je la réceptionne comme une offense, comme une patada al culo, ma chérie, una patada al culo qui me fait faire un salto de dix mètres en moi-même, qui ameute mon cerveau qui dormait depuis plus de quinze ans et qui me facilite de comprendre le sens des palabres que mon frère Josep a rapportées de Lérida. Alors quand on se retrouve en la rue, je me mets à griter (moi : à crier), à crier Elle a l'air bien modeste, tu comprends ce que ça veut dire ? Plus doucement pour l'amour du ciel, implore ma mère qui est une femme très éclipsée. (p. 13)

(6) je me raccorde (moi : je me rappelle), je me rappelle brusquement (p. 13)

Dans (6), la succession immédiate de part et d'autre de la parenthèse fait apparaître la différence de statut sémiotique d'éléments linguistiques. Ceux-ci constituent tous du DD, lequel relève de l'autonymie, selon le cadre théorique développé par Jacqueline Authier-Revuz dans lequel s'inscrit cet article. Le segment de DD de Montse se définit bien comme de l'autonymie insérée dans un contexte de ce que Jacqueline AuthierRevuz (2004) décrit comme Représentation du Discours Autre (dorénavant RDA), contexte par lequel il accède indirectement à la référence ; on peut faire l'hypothèse complémentaire qu'il inclut ensuite de la modalisation autonymique interlocutive allusive dans la reprise de "je me rappelle ", sous-entendant une possible glose en " comme tu dis ». Quant à la parenthèse elle-même, elle relève de l'autonymie renvoyant au mot comme forme linguistique ayant cette fois un référent virtuel : elle pourrait être glosée par « on dit/il faut dire: je me rappelle ».

On peut noter par ailleurs que tous les éléments de (5) qui ne relèvent pas du français académique ne sont pas pour autant l'objet d'un commentaire métalinguistique : ni le segment en espagnol (visiblement hétérogène), ni les calques de l'espagnol (palabres) ou les approximations (éclipsée), ni la construction avec la préposition en où le français préfèrerait dans ne suscitent de commentaire du fait de leur caractère facilement interprétable, à la différence éventuelle de griter (francisation de l'espagnol gritar). Le commentaire métalinguistique se limite ici à une forme minimale, la simple insertion d'un segment de DD par le recours aux parenthèses qui en opérant le changement de voix vaut pour un implicite « on ne dit pas à griter mais à crier » et invite à la « rature ». Forme minimale, mais efficace puisque la substitution est immédiatement effectuée à la reprise du DD de Montse.

18 Autre manifestation de ce caractère minimaliste du commentaire métalinguistique lié à l'interlocution, la glose peut porter non pas sur la totalité du mot jugé fautif mais sur un segment, comme dans le cas du passage de stomaqué à estomaqué. Cette réduction au strict minimum du commentaire a alors pour effet de déclencher un questionnement en retour, du fait de l'opacité produite par le recours à l'autonymie sur un segment inférieur à l'unité du mot :

(7) je n'en revenais pas, dit ma mère, j'étais complètement stomaquée, es dis-je, quoi es? estomaquée, j'étais estomaquée devant tant de riquesses. (p. 114) 

avant que celui-ci ne reprenne sa narration initiale : il relève d'abord de la rectification linguistique qui procède ici non plus par rature mais par ajout, quand le deuxième commentaire, interrogatif, témoigne du dysfonctionnement dans l'échange (situation c) évoquée par Jakobson).

A l'inverse, ce sont les lacunes lexicales ponctuelles de la mère en français qui peuvent déclencher son commentaire métalinguistique. Les mots absents du vocabulaire de Montse lui sont alors suggérés par la narratrice. Dans les exemples ci-dessous, le commentaire porte sur la forme ou l'existence même du mot, dans un rapport à la langue systématiquement montré comme ludique :

(8) Car mon frère, ma chérie, n'était pas un ..., couard dis-je, tu me fais rire avec tes mots increibles, me dit ma mère. (p. 178)

(9) une rue qui est en côte comme ça, me dit ma mère en inclinant sa main, un raidillon dis-je, tu inventes des mots maintenant? dit ma mère que ce mot amuse. (p. 52)

Si dans ces deux exemples, la lacune est du côté de celle à qui le mot fait défaut, on rencontre également le cas où le commentaire métalinguistique consiste en une glose apportée à un commentaire absent, celui-ci restant en creux mais trouvant un écho dans la glose qu'il déclenche. C'est le cas dans cet autre passage de DD entre Montse et sa fille où n'est pas explicitée la rectification apportée par la narratrice, seule subsiste sa trace dans la réponse :

(10) je me rappelle brusquement que je souffre d'un panadis au pouce et que j'ai le doigt bandé, panaris si tu veux, mais ne me rectifie pas à chaque mot sinon j'y arriverai jamais. (p. 14)

Le commentaire métalinguistique prend ici la forme de l'acceptation conditionnelle du vouloir dire ainsi de l'interlocutrice; de celui-ci ne subsiste matériellement dans le texte que l'écho qui en est fait par l'enregistrement de la rectification, aussitôt suivi de réticence.

De même, le commentaire métalinguistique qui intervient dans un second temps, dans une parenthèse en $\mathrm{DD}$, permet d'interpréter rétroactivement le segment qui précède et contient l'élément déclencheur du commentaire comme étant du Discours Indirect Libre (DIL). La bivocalité s'y fait percevoir dans la trace d'une prononciation erronée :

(11) Elle mange du veurre pour la première fois au petit déjeuner (on dit veurre ou beurre ma chérie? me demande ma mère qui confond les deux sons, je sais jamais).

(p. 121)

Dans ces différents exemples, on constate que la narration s'attache tout autant à parler du souvenir de 1936 qu'à interroger ou commenter les pratiques discursives qu'elle suscite, selon un mouvement de réflexivité insistant, même si celui-ci semble ne porter que sur des détails.

D'autres configurations avec glose sont encore mises en œuvre par le texte : ainsi des considérations sur le caractère approprié du mot de l'autre langue, mot français découvert dans l'échange interlocutif et qui soulève l'enthousiasme du récepteur par la prise en compte de la littérarité ou du signifiant, ou mot espagnol maintenu en français pour des raisons de force et de pertinence. Il peut s'agir soit d'un commentaire déclenché par l'échange dialogal (12), soit de boucles réflexives à l'intérieur de la narration (13), (14), soit de configuration (15) pour laquelle l'interprétation hésite entre commentaire métalinguistique apporté en réponse à une intervention de la narratrice 
qui traduit un mot de sa mère ou boucle réflexive au sein du DD de Montse. L'hésitation est autorisée par la ponctuation à laquelle L. Salvayre recourt pour introduire du DD ; les virgules à rôle démarcatif ne se distinguent pas ici de virgules juxtaposant des syntagmes attribuables à un même énonciateur :

(12) Et si son enthousiasme s'était singulièrement refroidi depuis les Jours Inoubliables, si son idée de la révolution s'était entachée d'une ombre dont la surface ne cessait de s'épandre (moi : réduite à une peau de chagrin, ma mère : que cette expression est belle!) (p. 145)

(13) Les jours passèrent, les règles ne venaient pas, et Montse dut admettre qu'elle était bel et bien embarazada, en espagnol le mot est plus parlant, (p. 151)

(14) Disons qu'ils éprouvèrent l'un pour l'autre de l'amistad (ma mère me dit qu'en espagnol le mot a davantage de panache, soit). (p. 225-226)

(15) Et dans ce jaleo, dans ce brouhaha, quel mot formidable ma chérie !, (p. 115)

On note que s'opère alors un renversement des rôles d'expert linguistique par rapport à la répartition mise en scène dans les exemples vus jusqu'à présent : c'est ici Montse, par sa différence langagière, qui est à même de porter les jugements sur les mots, jugements qui sont suivis par la narratrice.

métalinguistique (soit métiscursive dans léchange dialogat, métalinguistique (soit métadiscursive dans l'échange dialogal, soit métaénonciative en cas de boucle réflexive) explicitant un point de vue sur la forme langagière surgie en discours. A côté de ces configurations, on constate également la présence de gloses en $s i$ j'ose dire qui ponctuent le DD de Montse, non pour marquer l'hésitation sur la forme de langue ou l'évaluer en termes de beauté ou de correction, mais pour marquer une suspension irréalisante du dire. Si la glose relève bien toujours du métalinguistique entendu au sens large, plus spécifiquement du métaénonciatif, elle intervient moins pour souligner une forme erronée que pour signaler ce qui est donné comme une coquetterie langagière (une audace :j'ose) par Montse et s'apparente même à une forme de manifestation de distinction langagière semble-t-il ${ }^{4}$ :

(16) Une petite anisette, ma Lidia. Par les temps qui galopent, c'est une précaution qui n'est pas, si j'ose dire, surnuméraire. (p. 279)

(17) Toutes ces choses (dit ma mère) étaient le cadeau de mes soucis et je m'en tamponnais l'œil, si j'ose dire. (p. 162)

(18) Je n'arrivais pas réellement à l'embéléquer, si j'ose dire, me dit ma mère. (p. 205)

La glose s'accompagne dans ces exemples d'un double décalage : décalage par rapport à sa propre pertinence puisqu'elle est employée pour signaler une audace apparentée à une élégance de langage par son énonciatrice : identifiée par J. Authier-Revuz comme «l'un des tics métaénonciatifs les plus répandus qui soient »" la glose mériterait d'être à son tour glosée par un «comme on dit ", «comme disent les Français élégants ", mettant en scène et un effet de citation et un imaginaire linguistique ${ }^{6}$; décalage également car se trouve pointée par la glose une hétérogénéité à la place d'une autre, qui mériterait cette fois d'être glosée par un "c'est le cas de le dire», commentaire surplombant émanant alors de la narratrice. Cette hétérogénéité "décalée ", "décentrée " attire d'autant plus l'attention que dans le même énoncé d'autres formes, éloignées de la glose, témoignent encore de l'altérité (les temps qui galopent, le cadeau de mes soucis).

30 Ces formes, prises dans le DD de Montse, ne sont pas représentées comme telles par leur énonciatrice. Mais la présence d'une glose à proximité - glose qui 1) déclenche un contexte métalinguistique d'un autre ordre et 2) est susceptible d'appeler une glose qui 
pointe un fait métadiscursif - vient se combiner avec le contexte de DD qui est le leur : par définition forme métadiscursive, le DD relève bien de la Représentation de Discours Autre, de façon globale pour le segment autonyme. L'hypothèse est donc la suivante : le métalinguistique «_englobant» du DD vient jouer avec la présence effective de glose portant sur un autre objet et/ou signalant un autre fait d'hétérogénéité, pour susciter sur ces formes de fragnol un effet métalinguistique portant sur leur non coïncidence. Celle-ci est alors non plus seulement manifeste, ou présente, mais bien montrée par la convergence de ce qui devient des indices (et non pas des marques, car les gloses marquent explicitement ici autre chose).

31 L'hypothèse d'un effet métalinguistique s'appuie ainsi sur la prise en compte de configurations de DD qui n'ont d'autre particularité que d'intervenir pour représenter un discours autre à l'intérieur duquel il est aisé de repérer des formes de fragnol sans que celles-ci soient nécessairement assorties d'une glose spécifique contrairement aux exemples vus au début de l'étude :

(19) Montse, Rosita, Josep et Joan arrivent le soir du $1^{\text {er }}$ août dans la grande ville catalane où les milices libertaires se sont emparées du pouvoir. Et c'est la plus grande émotion de leur vie. Des heures inolvidables (me dit ma mère) et dont le raccord, le souvenir ne pourra jamais m'être retiré, nunca, nunca, nunca. (p. 110)

(20) Tu l'as comprendi ma chérie, me dit ma mère, dona Pura, dans sa rigidesse et sa rancœur de catholique offendée, était une Sainte Femme, assidue à toutes les messes, et dont le cœur saignait de voir une partie de l'Europe tomber dans le matérialisme, elle qui dédiquait tous ses efforts au perfectionnement de son âme par l'aplastement de toutes les voluptés terrestres. (p. 86)

(21) On s'est perdu en suppositions (dit ma mère) avec cette psychologie à deux balles comme tu dirais dont les gens s'encapricent quand ils sont privés des distractions élémentaires. (p. 36)

Si le DD, par définition, relève bien du métalinguistique au sens général, dans la mesure où il constitue l'un des modes de RDA et fait jouer l'autonymie, se surajoute dans ces configurations un effet de surlignement implicite de l'altérité langagière par la combinaison de l'incise de parole et de la concentration des formes non académiques. La présence de commentaires métalinguistiques qui interrogent la forme même du mot n'est bien sûr pas exclue. La différence réside alors dans le niveau d'assignation de la représentation: assignable à l'énonciatrice du DD pour la glose, quand l'effet métalinguistique est attribuable à la narratrice qui met en scène le DD :

(22) Josep s'en va sans arrepentiment (dit ma mère). Il n'a jamais pensé prender la direction $\mathrm{du}$ village, il ne galope pas derrière le pouvoir, et les vieux paysans s'équivoquent qui lui ont prêté l'intention de faire le cabot. A la différence de Diego, qui a, comme tu dirais, les dents longues, et dont les palabres et les actes semblent servir un gol secret, Josep est un cœur pur, ça existe ma chérie, ne te ris pas, Josep est un caballero, si j'ose dire, il aime régaler, est-ce que régaler est français ? Il s'est dédiqué à son rêve avec toute sa juventud et toute sa candeur, (p. 79)

Intermédiaires entre un pôle marqué par une glose et un pôle non marqué (de l'ordre du simplement manifeste), certains contextes de DD semblent pouvoir ainsi jouer le rôle d'embrayeurs d'effets métalinguistiques. Ils explicitent dans leur propre configuration la mention d'un dire qu'il s'avère nécessaire de montrer comme autre dans la mesure où les formes linguistiques citées, pour une partie d'entre elles, sont fautives au regard de la norme. On a noté dans ces exemples la relative concentration des formes de fragnol. C'est ainsi un rapport à la langue, pointé comme fautif (mais non condamné), qui est mis en scène, rapport dont la justification est systématiquement liée à la pratique hésitante du français par une locutrice d'origine étrangère. 

représenté et manifeste sont sollicitées aussi bien typographie, graphie, formes de RDA (DD), dans des configurations qui, de manières plus ou moins discrètes, insistent sur des formes sans nécessairement leur adjoindre un commentaire systématique. Dès lors se pose la question : la littérature, ou du moins celle qui se construit en interrogeant sa pratique discursive et son rapport à la langue, ne serait-elle pas un objet particulièrement propice à la mise en œuvre d'un effet métalinguistique?

\section{BIBLIOGRAPHIE}

Salvayre L. (2014). Pas Pleurer. Paris : Seuil. 
Salvayre L. (2015). Défense et illustration du fragnol. In Bienvenue !, 34 auteurs pour les réfugiés. Paris : Points. 153-156.

Authier-Revuz J. (1995 (2012)) Ces mots qui ne vont pas de soi. Boucles réflexives et non-coïncidences du dire. Limoges : Lambert-Lucas.

Authier-Revuz J. (2004). La représentation du discours autre : un champ multiplement hétérogène. In Lopez-Munoz J.-M., Marnette S., Rosier L. (eds), Le discours rapporté dans tous ses états : question de frontières, (actes du colloque Bruxelles 8-11 novembre 2001). Paris : L'Harmattan. 35-53.

Bres J., Mellet S. (2009). Langue française 163. Paris : Larousse.

Houdebine A.-M. (éd.) (2002). L'imaginaire linguistique. Paris : L'Harmattan.

Houdebine A.-M. (2002). « L'imaginaire linguistique : un niveau d'analyse et un point de vue théorique ». In Houdebine A.-M. (éd.), L'imaginaire linguistique. Paris : L’Harmattan. 9-21.

Houdebine-Gravaud A.-M. (1995). L'Unes langue. In Éloy J.-M. (éd.), La qualité de la langue ? Le cas du français. Paris : Honoré Champion. 95-121.

\section{NOTES}

1. Lydie Salvayre, Pas pleurer, 2014, désormais PP.

2. Le roman de Lydie Salvayre s'appuie sur les articles écrits par Bernanos pendant son séjour aux Baléares (1934-1937), réunis ensuite sous le titre Les grands Cimetières sous la lune et publiés aux éditions Plon en 1938. Les éditions du Castor Astral/Points ont réédité l'ouvrage en 2014, année de la publication de PP.

3. Authier-Revuz (1995(2012): 612)

4. Cf. l'éloge du fragnol dans «Défense et illustration du fragnol» (Salvayre 2015) qui prend comme illustration des mots savants la glose «si j'ose dire »: «Une langue éloignée de tout principe hiérarchique, mots savants et grossiers aimés d'un même cœur, je t'en foutrais si j'ose dire. » (p. 156)

5. Authier-Revuz (1995(2012): 635)

6. Au sens des normes subjectives définies par Houdebine (2002).

7. Cf. Bres et Mellet (2009) qui définissent par exemple un marqueur dialogique comme étant «un morphème dont le signifié en langue programme la signification dialogique ». Le redoublement graphique ne répond en rien au critère de stabilité ni à l'identité de morphème.

\section{RÉSUMÉS}

L'article interroge les limites du métalinguistique, en analysant le continuum possible entre des formes avec commentaire explicite et des segments sans commentaire qui déclenchent cependant une interprétation métalinguistique. Cet «effet métalinguistique "sera observé à partir du roman de Lydie Salvayre, Pas pleurer. 
The article questions the limits of metalinguistics, analyzing the possible continuum between forms with explicit commentary and segments without commentary that trigger a metalinguistic interpretation. This "metalinguistic effect" will be observed from Lydie Salvayre's novel, Pas pleurer.

INDEX

Mots-clés : métalinguistique; effet métalinguistique; méta énonciation; discours direct; dialogisme

Keywords : metalinguistic; metalinguistic effect; meta enunciation; direct speech; dialogism

\section{AUTEUR}

\section{CATHERINE RANNOUX}

Université de Poitiers, FORELLIS EA 3816 